Artículo

\title{
Variabilidad genética y criterios de selección del rendimiento y los componentes en trigos harineros de temporal
}

\author{
Eduardo Espitia Rangel ${ }^{1}$ \\ Eliel Martínez Cruz ${ }^{1 \S}$ \\ Héctor Eduardo Villaseñor Mir ${ }^{1}$ \\ René Hortelano Santa Rosa ${ }^{1}$ \\ Agustín Limón Ortega ${ }^{1}$ \\ Azalia Lozano Grande ${ }^{1}$
}

${ }^{1}$ Campo Experimental Valle de México-INIFAP. Carretera Los Reyes-Texcoco km 13.5, Coatlinchán, Texcoco, Estado de México, México. CP. 56250. (espitia.eduardo@inifap.gob.mx; villasenor.hector@inifap.gob.mx; hortelano.rene@inifap.gob.mx; limón.agustin@inifap.gob.mx; lozanoazalia@gmail.com; rodriguez.maria@inifap.gob.mx).

${ }^{\S}$ Autor para correspondencia: martinez.eliel@inifap.gob.mx.

\section{Resumen}

El conocimiento de la variabilidad genética y heredabilidad del rendimiento y sus componentes dentro del fito mejoramiento permite optimizar el proceso de selección. El objetivo de la investigación fue determinar la variabilidad genética del rendimiento y de los componentes, así como sus correlaciones para identificar criterios de selección para mayor productividad de grano en trigo harinero. Se utilizaron 19 genotipos, sembrados en el ciclo primavera-verano en siete localidades de temporal en los Valles Altos de México. El diseño experimental fue alfa látice con dos repeticiones en cada ambiente. La unidad experimental fue de cuatro surcos de $3 \mathrm{~m}$ de longitud con una separación de $30 \mathrm{~cm}$. Las variables evaluadas fueron: días a madurez, altura de planta, granos por espiga, número de espigas y granos por metro cuadrado, así como índice de cosecha, peso de mil granos y rendimiento de grano. Los mayores coeficientes de variación genética 20.8 y 18.4 fueron para rendimiento de grano y granos por espiga, respectivamente. Pero las variaciones genéticas y herededabilidades más altas las presento el peso de mil granos y granos por espiga lo cual facilita la selección de estos caracteres. El rendimiento presentó correlaciones significativas con número de granos por metro cuadrado, granos por espiga, número de espigas e índice de cosecha con valores de $0.92,0.57,0.54$ y 0.54 , respectivamente, que se pueden utilizar como criterios de selección para la productividad. Para este grupo de genotipos es necesario incrementar la variabilidad genética para espigas por metro cuadrado y peso de mil granos. Se propone a Don Carlos M2015, 6, 11 y 8 como fuentes genéticas para incrementar el peso de grano, número de granos por espiga, mayor número de espigas y granos por metro cuadrado para el rendimiento.

Palabras clave: criterios de selección, coeficiente de variación genética, componentes del rendimiento, heredabilidad, variabilidad genética.

Recibido: enero de 2021

Aceptado: marzo de 2021 


\section{Introducción}

El rendimiento promedio nacional de trigo harinero (Triticum aestivum L.) en condiciones de temporal en México fue de $2.5 \mathrm{t} \mathrm{ha}^{-1}$ con una superficie sembrada de 71193 ha (SIAP, 2020) en 2019. Sin embargo, se estima que su superficie sembrada puede incrementarse hasta en 300000 ha de tal manera que dicha producción puede ser una opción para disminuir las importaciones de este cereal las cuales fueron de alrededor de 4.2 millones de toneladas en 2016 (Canimolt, 2016). Una condición para que el cultivo tenga mayor aceptación entre los productores es incrementar su rentabilidad mediante el uso de variedades con mayor potencial de rendimiento.

Por esta razón uno de los principales objetivos del programa de mejoramiento de trigo de temporal del Instituto Nacional de Investigaciones Forestales, Agrícolas y Pecuarias (INIFAP) en México es la liberación de variedades que conjunten alto potencial de rendimiento (Villaseñor et al., 2018), tolerancia a enfermedades foliares (Rodríguez-García et al., 2019) y que cumplan con la calidad demandada por la industria (Hortelano et al., 2016).

Para el caso del mejoramiento genético para mayor potencial de rendimiento en condiciones de riego en México se han incorporado algunos criterios fisiológicos de selección como el peso de mil granos (Lopes et al., 2012), índice de cosecha y biomasa aérea (Lopes y Reynolds, 2011), de tal manera que se han tenido ganancias anuales en el rendimiento bajo riego restringido de $1.35 \%$ (Paquini et al., 2016), en buena medida debido también al uso intensivo de insumos y se menciona que se ha llegado a un tope por lo que es necesario buscar nuevas estrategias de mejoramiento para mantener estos avances.

En este contexto el programa de trigo de temporal es necesario la incorporación de diferentes herramientas dentro de estas las variables fisiológicas como criterios que ayuden a la selección de genotipos de mayor potencial de rendimiento. Como parte de estas estrategias se menciona el uso de los componentes del rendimiento para la implementación de esquemas de selección múltiple o indirecta del rendimiento. Para ello se requiere del conocimiento de la variabilidad genética local de los materiales o poblaciones base pues de ello depende la respuesta a la selección.

La variabilidad genética es expresada en medidas de dispersión como el rango, varianzas fenotípica y genotípica, sin embargo, el coeficiente de variación genética es el mejor parámetro para tal propósito (Ehdaie y Waines, 1989). Al respecto, Fellahi et al. (2013) reportaron valores de varianza fenotípica, varianza genética y coeficiente de variación genética para altura de planta de 98.33, 64.26 y 10.37, respectivamente; para peso de mil granos $15.05,9.09$ y 9.84 ; para granos por espiga $71.91,40.48$ y 5.17 ; para índice de cosecha $10.05,3.93$ y 5.17, así como para rendimiento de grano de $29.39,5.2$ y 9.84 .

El estrés por un agente biótico puede modificar los componentes del rendimiento, como reducir el número de espigas fértiles por unidad de área y el número de granos por espiga (Abayomi y Wright, 1999) y es reconocido que el llenado de grano es influenciado negativamente por altas temperaturas y sequía durante la maduración (Chmielewski y Kohn, 2000). El rendimiento del trigo se analiza a menudo en términos de los componentes del rendimiento: espigas por área, granos por espiga o tamaño de grano, así como entre sus correlaciones. Sin embargo, las compensaciones entre ellos es la principal barrera para mejorar el rendimiento utilizando este enfoque (Slafer et al., 2014). 
Por lo que el objetivo de esta investigación fue determinar la variabilidad genética del rendimiento y de sus componentes, así como sus correlaciones para identificar criterios que permitan aumentar la respuesta a la selección para rendimiento de grano en trigo harinero (Triticum aestivum L.) para temporal en los Valles Altos de la Mesa Central de México.

\section{Materiales y métodos}

Se utilizaron 19 genotipos de trigo harinero (Cuadro 1). Cinco corresponden a las variedades comerciales: Romoga F96, Tlaxcala F2000, Triunfo F2004, Altiplano F2007 y Don Carlos M2015. Las cuales han sido recomendadas para su siembra en diferentes ambientes de temporal por el INIFAP en diferentes periodos. Por otro lado, las 14 líneas restantes son los genotipos elite del programa de mejoramiento, las cuales presentan buen potencial de rendimiento, tolerancia a enfermedades y la calidad panadera demandada por la industria nacional. Por lo que este grupo de genotipos conjunta lo más sobresaliente del programa de mejoramiento genético de trigo harinero de temporal del INIFAP en México y las variedades comerciales se incluyen como testigos.

Cuadro 1. Genotipos de trigo harinero incluidos en el estudio de la variabilidad y asociaciones genéticas.

\begin{tabular}{|c|c|}
\hline Número & Genotipo \\
\hline 1 & $\begin{array}{l}\text { ROH//CENT.BLANCO/SNP/7/FAHAD_4/FARAS_ } \\
\text { 1/5/274/320//BGL/3/MUSX/LYNX }\end{array}$ \\
\hline 2 & ALHE/JUCHI \\
\hline 3 & PFAU/SERI.1B//AMAD/3/WAXWING/4/BABAX/LR42//BABAX*2/3/KURUKU \\
\hline 4 & Tlaxcala F2000 \\
\hline 5 & Romoga F96 \\
\hline 6 & GAVIA/ROM/GAL//BAR/MILAN/3/NANA \\
\hline 7 & GAV/ROM/GALV//BARC \\
\hline 8 & NAH/3/ALHE \\
\hline 9 & WBLL1/KAMB1//REB/3/BAR//GAV/GAL/4/NAH \\
\hline 10 & Don Carlos M2015 \\
\hline 11 & KA/NAC//TRCH/3/DANPHE \#1 \\
\hline 12 & $\mathrm{PRL} / 2 * \mathrm{REB} / 3 / \mathrm{BAR} / / \mathrm{GAV} / \mathrm{OCOR} / 4 / \mathrm{TRI}$ \\
\hline 13 & Gavia/ROM/GAL//BAR/MILAN/3/NANA \\
\hline 14 & Gavia/ROM/GAL//BAR/MILAN/3/NANA \\
\hline 15 & Altiplano F2007 \\
\hline 16 & ROH//CENT.Blanco/SNP/7/FAHAD_4/FARAS_1/5/274/320//BGL/3/MUSX/LYNX \\
\hline 17 & Monr/NANA \\
\hline 18 & $\mathrm{BAX} * 2 / \mathrm{PRL} / / \mathrm{TLAX} / 3 / \mathrm{NANA}$ \\
\hline 19 & Triunfo F2004 \\
\hline
\end{tabular}


Los genotipos se sembraron durante el ciclo primavera/verano (PV) en siete ambientes de temporal conformados de la siguiente manera: Soltepec, Tlaxcala; Juchitepec (2 fechas), Tlalmanalco, Coatepec, Chapingo y Santa Lucía, Estado de México. Las localidades de Santa Lucía de Prías y Chapingo se caracterizan por ser de clima templado subhúmedo $\mathrm{C}$ (Wo) (W) b(y)g. Chapingo se sitúa a $19^{\circ} 13^{\prime}$ latitud norte (LN) y $98^{\circ} 51^{\prime}$ longitud oeste (LO) a $2250 \mathrm{msnm}$ y Santa Lucía se localiza a $19^{\circ} 44^{\prime} \mathrm{LN}$ y $98^{\circ} 87^{\prime} \mathrm{LO}$ a $2260 \mathrm{msnm}$. Juchitepec y Coatepec se clasifican como de clima templado húmedo, Juchitepec se ubica a $2571 \mathrm{msnm}$ entre los $19^{\circ} 06^{\prime} \mathrm{LN}$ y $98^{\circ} 53^{\prime} \mathrm{LO}$ y Coatepec a $2320 \mathrm{msnm}$.

Sus coordenadas geográficas de Soltepec, Tlaxcala se localiza en las coordenadas $19^{\circ} 04^{\prime}$ "00" y

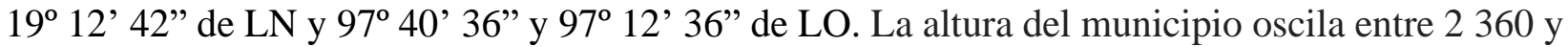
$2920 \mathrm{msnm}$. El municipio de Tlalmanalco, Estado de México se localiza en las coordenadas geográficas $19^{\circ} 80^{\prime} 48^{\prime \prime}$ y $19^{\circ} 15^{\prime} 43^{\prime \prime}$ de LN y $98^{\circ} 37^{\prime} 58^{\prime \prime}$ y $98^{\circ} 51^{\prime} 20^{\prime \prime}$ de LO con una altitud media de 2400 m (García, 1981).

El diseño experimental fue Alfa látice con dos repeticiones en cada ambiente. La unidad experimental fue de cuatro surcos de $3 \mathrm{~m}$ de longitud con una separación de $30 \mathrm{~cm}$. La densidad de siembra fue de $120 \mathrm{~kg} \mathrm{ha}^{1}$, se fertilizó con la fórmula 40-20-00, aplicando todo el $\mathrm{N}$ y todo el $\mathrm{P}$ a la siembra. Como fuente de fertilización se utilizó urea $\left(\mathrm{CO}\left(\mathrm{NH}_{2}\right)_{2}\right)$ con $46 \%$ de $\mathrm{N}$ y superfosfato de calcio triple $\left(\mathrm{Ca}\left(\mathrm{H}_{2} \mathrm{PO}_{4}\right)_{2}\right)$ con $46 \%$ de $\mathrm{P}_{2} \mathrm{O}_{5}$. El control de malezas de hoja ancha y angosta se realizó con Esteron $47^{\circledR}$ y Topik $24 \mathrm{EC}^{\circledR}$, respectivamente.

Las variables evaluadas en la unidad experimental fueron: días a madurez (DM) que se determinó cuando $80 \%$ de la parte aérea de las plantas mostraban color paja. La altura de planta se midió desde la superficie del suelo hasta la punta de la espiga en cada unidad experimental. (AP, cm). El número de granos por espiga (GPE) que se determinó al contar y promediar el número de granos por espiga en 10 espigas completas. El número de espigas por metro cuadrado (EPMC) se determinó contando en cuatro hileras las plantas y dividirlo entre el área cosechada.

Previo a la cosecha se realizó un muestreo de 50 tallos para estimar el índice de cosecha (IC) el cual se calculó dividiendo el rendimiento de grano de los 50 tallos y el peso total de los 50 tallos. La cosecha de la parcela completa se realizó con una mini-combinada Wintersteiger ${ }^{\circledR}$ cuando la humedad del grano fue menor a $14 \%$ y se midió el rendimiento de grano (RENG) en $\mathrm{kg} \mathrm{ha}^{-1}$. Se tomó una muestra de 200 granos al azar de cada parcela para determinar el peso de mil granos (PMG). El número de granos por metro cuadrado (GPMC) se obtuvo mediante el producto de EPMC y GPE.

Los datos se analizaron mediante GLM del SAS (SAS Institute, 2002). Se realizó un análisis de varianza para ambientes y genotipos, se realizaron pruebas de Tukey $(p \leq 0.05)$ para la comparación entre medias de genotipos y ambientes. El coeficiente de variación genética se calculó mediante el cociente de la desviación estándar genética entre la media.

Coeficientes mayores a 20 se clasifican como de variabilidad genética alta, de 10 a 20 variabilidad genética intermedia y menores a 10 variabilidad baja. La heredabilidad se obtuvo al dividir la varianza genética entre la varianza fenotípica. Adicionalmente se obtuvieron las correlaciones simples mediante el procedimiento CORR de SAS. 


\section{Resultados y discusión}

En el Cuadro 2 se presentan las varianzas genéticas, varianzas ambientales y la de su interacción, así como del rendimiento de grano y de sus componentes. En donde se observó que las variables granos por espiga, peso de mil granos e índice de cosecha presentan mayor varianza genética, mientras que días a madurez, altura de planta, espigas por metro cuadrado, granos por metro cuadrado y rendimiento de grano fue mayor la varianza ambiental.

Lo anterior indica que las variables granos por espiga, peso de mil granos e índice de cosecha presentan una mayor variación entre genotipos lo cual indica mayor número de loci segregantes y por lo tanto mayor número de genotipos diferentes que se puedan seleccionar (Molina, 1992; Espitia et al., 2004).

Cuadro 2. Componentes de varianza para rendimiento y sus componentes de 19 genotipos de trigo evaluados en siete ambientes de temporal.

\begin{tabular}{cccccc}
\hline Variable & $\sigma^{2}{ }_{\text {LOC }}$ & $\sigma^{2}{ }_{\text {REP(SUBQ }}$ & $\sigma^{2}{ }_{\mathrm{G}}$ & $\sigma^{2}{ }_{\text {LOC }{ }^{*} \mathrm{G}}$ & $\sigma^{2}$ Error \\
\hline DAM & 132.76 & 1.21 & 7.63 & 1.12 & 0.36 \\
AP & 58.78 & 2.53 & 28.97 & 1.86 & 32.32 \\
EPMC & 1721.4 & 426.22 & 528.31 & 873.75 & 2848.8 \\
GPMC & 2096316.3 & 152122.2 & 1190995.6 & 1561923.2 & 1909841.9 \\
GPE & 8.17 & 1.1 & 29.02 & 0.22 & 36.38 \\
PMG & 5.03 & 5 & 13.36 & 1.91 & 7.06 \\
IC & $1 \times 10^{-3}$ & $3 \times 10^{-4}$ & $2 \times 10^{-3}$ & $1 \times 10^{-3}$ & $2 \times 10^{-3}$ \\
RENG & 258411.3 & 86054.4 & 182274.3 & 164532.3 & 190295.7 \\
\hline
\end{tabular}

$\mathrm{DM}=$ días a madurez; $\mathrm{AP}=$ altura de planta $\mathrm{GPE}=$ número de granos por espiga; $\mathrm{GPMC}=$ número de granos por metro cuadrado $; \mathrm{EPMC}=$ número de espigas por metro cuadrado; $\mathrm{IC}=$ índice de cosecha; $\mathrm{PMG}=$ peso de mil granos; $\mathrm{RENG}=$ rendimiento de grano; $\sigma^{2}=$ varianza; $\mathrm{LOC}=$ localidad; $\mathrm{REP}=$ repetición; $\mathrm{SUBQ}=$ subloque, $\mathrm{G}=$ genotipo.

En el Cuadro 3 se indican los parámetros genéticos para rendimiento y sus componentes evaluados. El coeficiente de variación genética más alto fue para el rendimiento de grano, pero con valores bajos de heredabilidad lo cual se explica en parte por su variación genética menor al 30\%. Los componentes del rendimiento granos por espiga, número de granos por metro cuadrado, índice de cosecha, peso de mil granos y número de espigas por metro cuadrado, presentaron coeficientes de variación genética intermedios.

Para peso de mil granos y número de granos por espiga los valores encontrados de heredibilidad concuerdan con los indicados por Sadras y Slafer (2012); Philipp et al. (2018) quienes reportaron heredabilidades de 0.6 y 0.59, respectivamente. Dichos valores se clasificaron como altos con base a lo indicado por Stanfield (1971). Lo anterior, se explica debido a que $62 \%$ de la varianza fenotípica correspondió a la varianza genética lo que indica que estas variables pueden mejorarse fácilmente porque su variación se debe principalmente a efectos genéticos. 
Cuadro 3. Parámetros genéticos para rendimiento y sus componentes en 19 genotipos de trigo evaluados en siete ambientes de temporal.

\begin{tabular}{ccccc}
\hline Variable & $\sigma^{2} \mathrm{P}$ & $\sigma^{2} \mathrm{G}$ & $\mathrm{h}^{2}$ & $\mathrm{CVG}$ \\
\hline Días a madurez & 133.03 & 7.63 & 0.05 & 2.24 \\
Altura de planta $(\mathrm{cm})$ & 69.46 & 28.97 & 0.41 & 6.1 \\
Espigas por metro cuadrado & 5870.17 & 528.31 & 0.09 & 10.37 \\
Granos por metro cuadrado & 5720203.6 & 1190995.6 & 0.2 & 17.15 \\
Granos por espiga & 45.43 & 29.01 & 0.63 & 18.42 \\
Peso de mil granos $(\mathrm{g})$ & 19.02 & 13.36 & 0.70 & 11.54 \\
Índice de cosecha $(\%)$ & $4 \times 10^{-3}$ & $2 \times 10^{-3}$ & 0.45 & 12.49 \\
Rendimiento de grano $\left(\mathrm{kg} \mathrm{ha}^{-1}\right)$ & 699293.7 & 182274.3 & 0.26 & 20.81 \\
\hline
\end{tabular}

$\sigma_{\mathrm{P}}^{2}, \sigma_{\mathrm{G}}^{2}, \mathrm{H}^{2}$; CVG; varianza fenotípica; varianza genética; heredabilidad y coeficiente de variación genética, respectivamente.

El índice de cosecha y altura de planta presentaron valores intermedios de heredabilidad, mientras que los valores más bajos los presentó rendimiento de grano, días a madurez y espigas por metro cuadrado. Para el caso del índice de cosecha su coeficiente de variación genética y heredabilidad permiten realizar selección, para incrementar su promedio de 0.33 a 0.58 dado que fue el valor máximo encontrado (Cuadro 4). Lo anterior, es necesario dado que índices mayores a 0.5 favorecen el potencial de rendimiento de acuerdo, a lo señalado por Reynolds et al. (2012); Zhang et al. (2016) quienes además indicaron la necesidad de incrementar la biomasa dado que es la fuente necesaria para incrementar continuamente el rendimiento.

Para el caso de número de granos por metro cuadrado y espigas por metro cuadrado se obtuvieron amplitudes de 16854 y 581.47 lo cual indica que es posible aplicar el mejoramiento genético para aprovechar su variabilidad (Cuadro 4). Con base en lo anterior, se debe incrementar la variabilidad genética para espigas por metro cuadrado, peso de mil granos e índice de cosecha. Mientras que se pueden aprovechar mediante la selección las características de número de granos por espiga y peso de mil granos debido a su alta variabilidad genética y heredabilidad para el grupo de genotipos estudiados.

Cuadro 4. Medias y rangos para rendimiento $y$ sus componentes en 19 genotipos de trigo evaluados en siete ambientes de temporal.

\begin{tabular}{lcccc}
\hline Variable & Media & Mínimo & Máximo & DS \\
\hline Días a madurez & 123.47 & 98 & 143 & 10.8 \\
Altura de planta $(\mathrm{cm})$ & 88.24 & 55 & 131 & 10.71 \\
Índice de cosecha & 0.33 & 0.09 & 0.58 & 0.07 \\
Espigas por metro cuadrado & 221.66 & 54.63 & 636.1 & 78.13 \\
Granos por metro cuadrado & 6363 & 1251 & 18105 & 2558 \\
Granos por espiga & 29.25 & 10.87 & 81.36 & 8.47 \\
Peso de mil granos $(\mathrm{g})$ & 31.69 & 16.13 & 45.89 & 5.54 \\
Rendimiento de grano $\left(\mathrm{kg} \mathrm{ha}^{-1}\right)$ & 2052 & 240 & 5458 & 911.65 \\
\hline
\end{tabular}

DS= desviación estándar. 
En el Cuadro 5 se presentan los promedios por genotipo para rendimiento y sus componentes. La línea experimental 6 superó en rendimiento a todos los genotipos evaluados, además presentó el segundo valor más alto de espigas por metro cuadrado y el más alto de número de granos por metro cuadrado, lo cual concuerda con Bustos et al. (2013) quienes indicaron que el número de granos es una de las variables más importantes para incrementar el rendimiento en trigo.

Cuadro 5. Medias por genotipo para rendimiento y sus componentes en trigos evaluados en siete ambientes de temporal.

\begin{tabular}{ccccccccc}
\hline Núm. genotipo & DAM & AP & EPMC & GPE & GPMC & PMG & IC & RENG \\
\hline 6 & 121.7 & 89.07 & 261.97 & 31.33 & 8197.3 & 35.56 & 0.36 & 2924.9 \\
Don Carlos M2015 & 128.91 & 109.71 & 165.9 & 42.34 & 7045.5 & 38.99 & 0.39 & 2637.1 \\
11 & 125.8 & 92.57 & 230.84 & 32.34 & 7483.3 & 35.64 & 0.35 & 2623.3 \\
16 & 122.4 & 82.93 & 178.66 & 38.62 & 7009.3 & 36.56 & 0.4 & 2588.4 \\
8 & 129.7 & 87.29 & 278.6 & 26.51 & 7513.6 & 31.79 & 0.29 & 2403.1 \\
1 & 126.2 & 91 & 217.96 & 32.6 & 6545.6 & 34.28 & 0.33 & 2277.9 \\
18 & 123.3 & 88.43 & 212.47 & 31.37 & 6738.4 & 33.13 & 0.34 & 2249.5 \\
17 & 121.36 & 89.5 & 192.75 & 34.02 & 6303.9 & 31.97 & 0.35 & 2231.9 \\
Triunfo F2004 & 121.73 & 85.36 & 239.72 & 25.64 & 6231.6 & 33.11 & 0.31 & 2129.9 \\
Romoga F96 & 122 & 86.64 & 244.09 & 33.49 & 8016.9 & 26.37 & 0.33 & 2107.6 \\
Tlaxcala F2000 & 123.82 & 86 & 253.81 & 28.39 & 7126.7 & 29.24 & 0.33 & 2105.9 \\
2 & 124.7 & 87.57 & 231.94 & 27.1 & 6283.2 & 30.91 & 0.32 & 1929.8 \\
7 & 124 & 86.14 & 250.79 & 25.39 & 5855.7 & 32.90 & 0.32 & 1929.6 \\
9 & 124.9 & 85.43 & 226.71 & 28.6 & 6545.6 & 27.23 & 0.32 & 1765.6 \\
13 & 121.73 & 86.57 & 211.81 & 26.04 & 5400.4 & 31.74 & 0.32 & 1698.4 \\
Altiplano F2007 & 121.73 & 87.07 & 173.96 & 26.76 & 4651.9 & 34.47 & 0.29 & 1574.1 \\
14 & 119.18 & 85.64 & 220.52 & 25.28 & 5504.1 & 28.50 & 0.3 & 1553.4 \\
3 & 123.9 & 82.93 & 227.89 & 26.44 & 5449 & 27.78 & 0.3 & 1508.9 \\
12 & 119.6 & 86.71 & 174.86 & 17.52 & 3125.1 & 23.50 & 0.19 & 754.4 \\
DSH & 3.97 & 7.9 & 82.03 & 8.92 & 2156.1 & 4.35 & 0.06 & 659.51 \\
\hline
\end{tabular}

$\mathrm{DAM}=$ días a madurez; $\mathrm{AP}=$ altura de planta, $\mathrm{cm} ; \mathrm{GPE}=$ número de granos por espiga; $\mathrm{GPMC}=$ número de granos por metro cuadrado; $\mathrm{EPMC}=$ número de espigas por metro cuadrado; $\mathrm{IC}=$ índice de cosecha; $\mathrm{PMG}=$ peso de mil granos $(\mathrm{g}) ; \mathrm{RENG}=$ rendimiento de grano $\left(\mathrm{kg} \mathrm{ha}^{-1}\right) ; \mathrm{DSH}=$ diferencia significativa honesta.

Por otro lado, la variedad testigo Don Carlos M2015 que ocupó el segundo lugar de rendimiento de grano mostró los valores más altos de granos por espiga y peso de mil granos, lo que concuerda con Feng et al. (2018) quienes mediante coeficientes de sendero encontraron que estas variables tuvieron la mayor relación con el rendimiento. Con base en lo anterior, se observó que ningún genotipo combinó los valores más altos de peso de mil granos y número de granos lo cual concuerda con lo indicado por Foulkes et al. (2011) que encontraron una relación lineal negativa entre estos caracteres. 
Por otro lado, el genotipo 10 y los cinco primeros lugares de mayor productividad de grano presentaron número de granos por metro cuadrado mayores a 7000 los cuales corresponde a los valores más altos, respecto al resto de los genotipos evaluados. Es importante destacar que el genotipo 10 identificado como la variedad comercial Don Carlos M2015 presentó valores mayores a 43 g de peso mil granos que concuerda con lo indicado por Buendía et al. (2019) quienes además mostraron que presentó valores altos para ancho y largo de grano respecto al resto de las variedades comerciales recomendadas para su cultivo de temporal en México.

Con base en lo anterior, Don Carlos M2015 puede ser una fuente genética que derive progenies de valores altos de peso de grano y de granos por espiga. Mientras que las líneas experimentales 6, 11 y 8 pueden aportar como progenitores al programa de mejoramiento progenies con mayor número de espigas y granos por metro cuadrado.

El rendimiento de grano presentó la correlación más alta y positiva con número de granos por metro cuadrado, así como correlaciones intermedias con número de granos por espiga, espigas por metro cuadrado e índice de cosecha (Cuadro 6). Lo anterior, concuerda con Bennett et al. (2012); Beche et al. (2014); García et al. (2014); Griffiths et al. (2015); Philipp et al. (2018) quienes reportaron correlaciones altas y positivas entre el número de granos y rendimiento. Por lo que se considera a este variable como la más importante para apoyar en la mejora del rendimiento de acuerdo con lo indicado por Philipp et al. (2018).

Cuadro 6. Correlaciones de Pearson entre rendimiento y sus componentes para 19 genotipos de trigo evaluados en siete ambientes de temporal, PV 2014.

\begin{tabular}{cccccccc}
\hline Variables & AP & IC & EPMC & GPMC & GPE & PMG & RENG \\
\hline DAM & $0.7^{* *}$ & $-7 \times 10^{-3} \mathrm{~ns}$ & $-0.16^{*}$ & $0.1 \mathrm{~ns}$ & $0.25^{* *}$ & $0.1 \mathrm{~ns}$ & $0.16^{*}$ \\
AP & & $0.15^{*}$ & $8 \times 10^{-3} \mathrm{~ns}$ & $0.34^{* *}$ & $0.45^{* *}$ & $0.27^{* *}$ & $0.4^{* *}$ \\
IC & & & $-0.1 \mathrm{~ns}$ & $0.41^{* *}$ & $0.69^{* *}$ & $0.59^{* *}$ & $0.54^{* *}$ \\
EPMC & & & $0.64^{* *}$ & $-0.2^{* *}$ & $-0.05 \mathrm{~ns}$ & $0.54^{* *}$ \\
GPMC & & & & $0.56^{* *}$ & $0.12 \mathrm{~ns}$ & $0.92^{* *}$ \\
GPE & & & & & $0.29^{* *}$ & $0.57^{* *}$ \\
PMG & & & & & & $0.45^{* *}$ \\
\hline
\end{tabular}

$\mathrm{ns},{ }^{*},{ }^{* *}=$ no significativo, significativo y altamente significativo. $\mathrm{DAM}=$ días a madurez; $\mathrm{AP}=$ altura de planta; GPE= número de granos por espiga; $\mathrm{GPMC}=$ número de granos por metro cuadrado; $\mathrm{EPMC}=$ número de espigas por metro cuadrado; $\mathrm{IC}=$ índice de cosecha; $\mathrm{PMG}=$ peso de mil granos; $\mathrm{RENG}$ = rendimiento de grano.

Así mismo coincide parcialmente con lo encontrado por Beche et al. (2014); Zhou et al. (2014) para la correlación entre índice de cosecha y rendimiento de grano, dichos autores reportaron correlaciones mayores a 0.9 superiores a las encontradas en este estudio, lo cual indica que dicha variable se puede usar indirectamente como criterio de selección para rendimiento de grano. A su vez se encontraron correlaciones positivas entre el índice de cosecha con granos por espiga y peso de mil granos. 
Lo que indica que ambos variables se pueden utilizar para seleccionar para rendimiento de grano lo que concuerda con lo indicado por Aisawi et al. (2015). Por otro lado, el número de granos por metro cuadrado se correlacionó positivamente con espigas por metro cuadrado por lo que este componente también puede utilizarse como criterio de selección para rendimiento de grano.

\section{Conclusiones}

Es necesario introducir, en el programa de mejoramiento de trigo de temporal del INIFAP, fuentes genéticas que permitan incrementar la variabilidad para espigas por metro cuadrado y peso de mil granos, mientras se pueden aprovechar mediante la selección las características de número de granos por espiga y peso de mil granos debido a su alta variabilidad genética y heredabilidad, específicamente en este grupo de genotipos. Para optimizar la discriminación por potencial de rendimiento se pude emplear indirectamente como criterio de selección principalmente el número de granos por metro cuadrado.

Se propone utilizar como progenitores dentro del plan de cruzamientos del programa de mejoramiento de trigo de temporal del INIFAP a los genotipos Don Carlos M2015 y las líneas experimentales 6, 11 y 8 como fuentes genéticas para incrementar el peso de grano, numero de granos por espiga, mayor número de espigas y granos por metro cuadrado, variables que favorecen el rendimiento de grano.

\section{Literatura citada}

Aisawi, K. A. B.; Reynolds, M. P.; Singh, R. P. and Foulkes, M. J. 2015. The physiological basis of the genetic progress in yield potential of CIMMYT spring wheat cultivars from 1966 to 2009. Crop Sci. 55(4):1749-1764.

Beche, E.; Benin G.; Da-Silva, C. L.; Munaro, L. B. and Marchese, J. A. 2014. Genetic gain in yield and changes associated with physiological traits in Brazilian wheat during the $20^{\text {th }}$ century. European J. Agron. 61(1):49-59.

Bennett, D.; Izanloo, A.; Reynolds, M.; Kuchel, H.; Langridge, P. and Schnurbusch, T. 2012. Genetic dissection of grain yield and physical grain quality in bread wheat (Triticum aestivum L.) under water-limited environments. Theor. Appl. Genet. 125(2):255-271.

Buendía-Ayala, B. L.; Martínez-Cruz, E.; Villaseñor-Mir, H. E.; Hortelano-Santa, R. R.; EspitiaRangel E. y Buendía-González, M. O. 2019. La incidencia de roya amarilla y la calidad industrial del grano y la masa en trigo harinero. Rev. Mex. Cien. Agríc. 10(1):143-154.

Bustos, D. V.; Hasan, A. K.; Reynolds, M. P. and Calderini, D. F. 2013. Combining high grain number and weight through a DH-population to improve grain yield potential of wheat in high-yielding environments. Field Crops Res. 145(4):106-115.

Canimolt. 2016. Cámara Nacional de la Industria Molinera de Trigo. Reporte estadístico 2015 con datos de 2016. http://canimolt.org/wp-content/uploads/2018/06/Canimolt2016190717.pdf.

Espitia, R. E.; Villaseñor, M. H. E.; Peña, B. R. J.; Huerta, E. J. y Limón, O. A. 2004. Calidad industrial de trigos harineros mexicanos para temporal. II. Variabilidad genética y criterios de selección. Rev. Fito. Mex. 27(1):41-47. 
Feng, F.; Han, Y.; Wang, S.; Yin, S.; Peng, Z.; Zhou, M.; Gao, W.; Xiaoxia, W.; Xiaoliang, Q. and Siddique, K. H. 2018. The effect of grain position on genetic improvement of grain number and thousand grain weight in winter wheat in North China. Frontiers Plant Sci. 9(2):1-9.

Foulkes, M. J.; Slafer, G. A.; Davies, W. J.; Berry, P. M.; Sylvester-Bradley, R.; Martre, P.; Calderini, D. F.; Griffiths, S. and Reynolds, M. P. 2011. Raising yield potential of wheat. (III) Optimizing partitioning to grain while maintaining lodging resistance. J. Exp. Bot. 62(2):469-486.

García, G. A.; Serrago, R. A.; González, F. G.; Slafer, G. A.; Reynolds, M. P. and Miralles, D. J. 2014. Wheat grain number: identification of favourable physiological traits in an elite doubled-haploid population. Field Crops Res. 168(10):126-134.

García, M. E. 1981. Modificaciones al sistema de clasificación climática de Köppen (para adaptarlo a las condiciones de la República Mexicana). $3^{\mathrm{a}}$ (Ed). Universidad Nacional Autónoma de México (UNAM). México, D. F. 252 p.

Griffiths, S.; Wingen, L.; Pietragalla, J.; García, G.; Hasan, A.; Miralles, D.; Calderini, D. F.; Ankleshwaria, J. B.; Waite, M. L.; Simmonds, J.; Snape, J. and Reynolds, M. 2015. Genetic dissection of grain size and grain number trade-offs in CIMMYT wheat germplasm. PloS One. 10(3):1-18.

Hortelano-Santa, R. R.; Espitia-Rangel, E.; Martínez-Cruz, E.; Villaseñor-Mir, H. E.; HuertaEspino, J. y Mariscal-Amaro, L. A. 2016. Productividad y calidad industrial de trigos harineros en relación con enfermedades. Agrociencia. 50(8):1027-1039.

Lopes, M. S. and Reynolds, M. P. 2011. Drought adaptive traits and wide adaptation in elite lines derived from resynthesized hexaploid wheat. Crop Sci. 51(4):1617-1626.

Lopes, M. S.; Reynolds, M. P.; Jalal-Kamali, M. R.; Moussa, M.; Feltaous, Y.; Tahir, I. S. A.; Barma, N.; Vargas, M.; Mannes, Y. and Baum, M. 2012. The yield correlations of selectable physiological traits in a population of advanced spring wheat lines grown in warm and drought environments. Field Crops Res. 128(3):129-136.

Philipp, N.; Weichert, H.; Bohra, U.; Weschke, W.; Schulthess, A. W. and Weber, H. 2018. Grain number and grain yield distribution along the spike remain stable despite breeding for high yield in winter wheat. PloS One. 13(10):1-17.

Reynolds, M.; Foulkes, J.; Furbank, R.; Griffiths, S.; King, J.; Murchie, E.; Parry, M. and Slafer, G. 2012. Achieving yield gains in wheat. Plant Cell Envir. 35(10):1799-1823

Rodríguez-García, M. F.; Rojas-Martínez, R. I.; Huerta-Espino, J.; Villaseñor-Mir, H. E.; ZavaletaMejía, E.; Sandoval-Islas, J. S. y Crossa-Hiriart, J. F. 2019. Genética de la resistencia a roya amarilla causada por Puccinia striiformis f. sp. tritici W. en tres genotipos de trigo (Triticum aestivum L.). Rev. Fito. Mex. 42(1):31-38.

Sadras, V. O. and Slafer, G. A. 2012. Environmental modulation of yield components in cereals: heritabilities reveal a hierarchy of phenotypic plasticities. Field Crops Res. 127(2):215-224.

SAS Institute. 2002. SAS/STAT User's Guide, Software versión 9.0. Cary, N. C. USA. 4424 p.

SIAP. 2020. Servicio de información Agroalimentaria y Pesquera. http://infosiap.siap.gob.mx: 8080/ agricola_siap_gobmx/AvanceNacionalSinPrograma.do.

Stanfield, W. D. 1971. Genética (teoría y 440 problemas resueltos). 2 (Ed). Serie Schaum, GrawHill, México, DF. 405 p.

Villaseñor, M. H. E.; Huerta Espino, J.; Hortelano-Santa, R. R.; Martínez-Cruz, E.; EspitiaRangel, E.; Solís-Moya, E.; Osorio-Alcalá, L. y Rodríguez-García, M. F. 2018. Valles F2015: nueva variedad de trigo para siembras de temporal en México. Rev. Mex. Cien. Agríc. 9(6):1315-1320. 
Zhang, Y.; Xu W.; Wang, H.; Dong, H.; Qi, X.; Zhao, M.; Fang, Y.; Gao, C. and Hu, L. 2016. Progress in genetic improvement of grain yield and related physiological traits of Chinese wheat in Henan Province. Field Crops Res. 199(12):117-128.

Zhou, B.; Sanz-Sáez, Á.; Elazab, A.; Shen, T.; Sánchez-Bragado, R.; Bort, J.; Serret, M. D. and Araus, J. L. 2014. Physiological traits contributed to the recent increase in yield potential of winter wheat from Henan Province, China. J. Integrative Plant Bio. 56(5):492-504. 